\title{
The Frontier of SGD and its Variants in Machine Learning
}

\author{
Juan Du ${ }^{1, a}$ \\ ${ }^{1}$ New Research and Development Center of Hisense, Qingdao 266071, China \\ adqxwpl@sina.com
}

Keywords: SGD, Stochsatic algorithms, optimization, iterative algorithm, machine learning

\begin{abstract}
Numerical optimization is a classical field in operation research and computer science, which has been widely used in the areas such as physics and economics. Although, optimization algorithms have achieved great success for plenty of applications, handling the big data in the best fashion possible is a very inspiring and demanding challenge in the artificial intelligence era. Stochastic gradient descent (SGD) is pretty simple but surprisingly, highly effective in machine learning models, such as support vector machine (SVM) and deep neural network (DNN). Theoretically, the performance of SGD for convex optimization is well understood. But, for the non-convex setting, which is very common for the machine learning problems, to obtain the theoretical guarantee for SGD and its variants is still a standing problem. In the paper, we do a survey about the SGD and its variants such as Momentum, ADAM and SVRG, differentiate their algorithms and applications and present some recent breakthrough and open problems.
\end{abstract}

\section{Introduction}

Optimization is an important tool in decision science and in the analysis of physical systems. In the simplest case, an optimization problem consists of a real objective function and a set of constraints. The goal of the problem is to find the maximum or minimum solution of the objective function in the feasible domain of the constraints. Formally speaking, the optimization problem can written as

$$
\begin{aligned}
& \quad \min _{x \in R^{d}} f(x) \\
& \text { s.t. } \\
& g_{i}(x) \leq 0, \quad \forall i \in \mathcal{E}, \\
& h_{j}(x)=0, \quad \forall j \in \mathcal{J},
\end{aligned}
$$

Where $f(x)$ is the objective function and $g_{i}(x)$ and $h_{j}(x)$ are the constraints.

It is a long story of the development of algorithms to solve the problem. In 1947, Dantzig[5] offered the first practical algorithm for linear programming which is known as simplex algorithm . However, it takes exponential time in the worst case. Later, Leonid Khachiyan proved that ellipsoid method can solve the linear programming in polynomial time. However, the ellipsoid method does not perform well in practice. The first practical polynomial time algorithm called Karmarkar's algorithm [1]. Actually, it is one of the interior point methods, which is a certain class of algorithms that can solve linear and nonlinear convex optimization problems. There are two common ways to design a interior point algorithm. One is called barrier method and the other is called primal-dual interior point algorithm.

Except the methods for general convex programming, for some specific settings, there are more effective algorithms. One useful setting is that the programming is unconstrained, in which the support domain of the variables are unconstrained. Although, it is more simplified version for the original problem, it is very common in the machine learning problems. Gradient descent (GD), which is also known as full batch gradient descent algorithm, is an implemented algorithm for the unconstrained problems. The iteration method of GD is pretty simple as follows.

$$
x^{t+1}=x^{t}-\gamma \nabla f\left(x^{t}\right)
$$


Where $\gamma$ is an adequately chosen step length? The algorithm achieves linear convergence when the initial estimate $x^{0}$ is close enough to the optimum and the step length $\gamma$ is sufficiently small. Here, linear convergence means that $\log \left(\frac{1}{\epsilon}\right) \sim t$ where $\epsilon$ represents the residual error. Methods based on Newton's method and inversion of the Hessian techniques can be better alternatives. Such methods converge in fewer iterations, but the cost of each iteration is higher. An example is the BFGS [11] method. Although these second order gradient descent methods usually achieve quadratic convergence, the second order differential may not exist or is very difficult to obtain. There are some techniques to avoid computing a Hessian matrix exactly. One common method is quasi-Newton method like BFGS.

There are some variants of BFGS, such as L-BFGS method, to handle the memory limited issues. The real world problems are too large to compute the full batch gradient descent each time. We should note that the real problem in machine learning has more specific characters. Usually, in the supervised learning setup, the objective function has the form of a sum, which is defined as follows.

$$
f(x)=\frac{1}{n} \sum_{i=1}^{n} f_{i}(x)
$$

Each summand function $f_{i}(x)$ is typically associated with the $i$-th observation in the data set. Usually, $f_{i}(x)$ is the value of the loss function at $i$-th example and $f(x)$ is called expirical risk. There are many machine learning problems has the form, such as

SVM: $f(w)=\lambda w^{2}+\sum \max \left\{0,1-y_{i} w^{T} \Phi\left(x_{i}\right)\right\}$ for $\Phi\left(x_{i}\right) \in R^{d}, y= \pm 1$

K-Means: $f(w)=\sum_{z_{i}} \min _{w_{1}, w_{2} \ldots w_{k}}\left(z_{i}-w_{j}\right)^{2}$ for $z_{i} \in R^{d}, w_{1}, \ldots w_{d} \in R^{d}$

Lasso: $f(w)=\lambda|w|_{1}+\sum\left(1-y_{i} w^{T} \Phi\left(x_{i}\right)\right)^{2}$ for $\Phi\left(x_{i}\right) \in R^{d}, y= \pm 1$

In this setting, the stochastic gradient descent algorithm performs much better. Instead of computing the gradient descent exactly, in each iteration, randomly pick one component $f_{i}(x)$ and do the following update:

$$
x^{t+1}=x^{t}-\gamma^{t} \nabla f_{i}\left(x^{t}\right)
$$

Note that the index $i$ is randomly picked in each iteration. Thus, in expectation, the SGD has the same performance as GD. There are plenty of works studying the performance of SGD in several different settings. For the non-smooth objective functions, the convergence rate of SGD is $O\left(1 / \varepsilon^{2}\right)$, and for the smooth functions, the convergence rate is $O(1 / \varepsilon)$.[4] [21]. The searching path of SGD is shown in Fig. 1 for an example (The sinuous vector path is called zig-zagging).

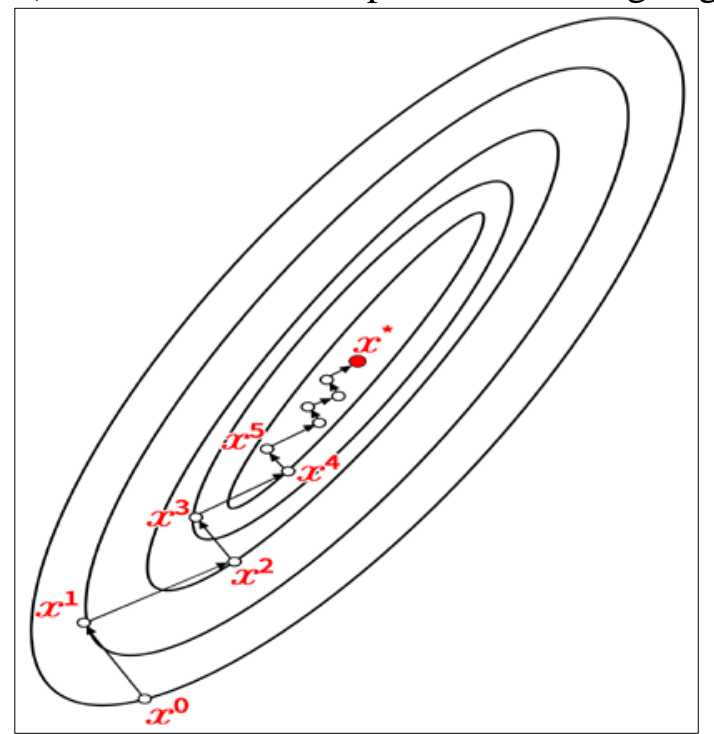

Fig. 1 The illustration of the searching path of SGD

Here is a detailed example.

( $x^{i}$ is the optimal result of the $i^{\text {th }}$ iteration) 
[Task 1]: The data of the 4 samples of function $F(X)=\sum_{j=1}^{n-1} \theta_{j} x_{j}+\theta_{n}\left(\forall X \in R^{(n-1)}\right)$ are listed in the Table 1 as below, please find the best simulated $F(X)$.

Table 1 Sample Set 01

\begin{tabular}{|l|c|c|c|}
\hline i(Sample No.) & $x_{1}^{i}$ & $x_{2}^{i}$ & $F^{i}\left(x_{1}^{i}, x_{2}^{i}\right)$ \\
\hline 1 & 2 & 1 & 11 \\
\hline 2 & 2 & 2 & 16 \\
\hline 3 & 3 & 1 & 14 \\
\hline 4 & 3 & 2 & 19 \\
\hline
\end{tabular}

Solution with algorithm SGD can be summarized as following:

Posit that the target linear function is $F\left(x_{1}, x_{2}\right)=\theta_{1} x_{1}+\theta_{2} x_{2}+\theta_{3}$.

If $\theta=\left(\begin{array}{c}\theta_{1} \\ \theta_{2} \\ \theta_{3}\end{array}\right), X=\left(\begin{array}{c}x_{1} \\ x_{2} \\ 1\end{array}\right)$, then $F\left(x_{1}, x_{2}\right)=\boldsymbol{X}^{\boldsymbol{T}} \boldsymbol{\theta}\left(\theta \in R^{3}, X \in R^{3}\right)$

[Iteration1]: Select a sample randomly from the sample set with 4 samples.

Posit that the sample randomly selected is sample 1: $\boldsymbol{X}_{\boldsymbol{s}_{\mathbf{1} \times \mathbf{3}}}^{\mathbf{1}}=\left(\begin{array}{lll}\mathbf{2} & \mathbf{1} & \mathbf{1}\end{array}\right), \boldsymbol{F}_{\boldsymbol{s}_{\mathbf{1} \times \mathbf{1}}}^{\mathbf{1}}=\mathbf{1 1}$

The deviation function is: $V(\theta)_{1 \times \mathbf{1}}^{1}=F_{s}^{1}-F\left(X_{s}^{1}\right)=11-\left(2 \theta_{1}+\theta_{2}+\theta_{3}\right)$

The loss function (Target of the optimization) $J(\theta)_{1 \times 1}^{1}=\frac{1}{2}\left(11-\left(2 \theta_{1}+\theta_{2}+\theta_{3}\right)\right)^{2}$

The 1st order gradient of $J(\theta): G(\theta)_{3 \times 1}^{1}=-\left(X_{s}^{1}\right)^{T} V(\theta)^{1}=\left(\begin{array}{c}4 \theta_{1}+2 \theta_{2}+2 \theta_{3}-22 \\ 2 \theta_{1}+\theta_{2}+\theta_{3}-11 \\ 2 \theta_{1}+\theta_{2}+\theta_{3}-11\end{array}\right)$

Posit that the initial value of $\boldsymbol{\theta}$ is $\boldsymbol{\theta}^{\mathbf{1}}=\left(\begin{array}{l}0 \\ 0 \\ 0\end{array}\right)$, then the initial $G(\theta)^{1}=\left(\begin{array}{l}-22 \\ -11 \\ -11\end{array}\right)$.

The deviation function is: $V(\theta)^{1}=11, J(\theta)^{1}=60.5$

If $\eta^{1}$ is set to be 0.1 , then $\boldsymbol{\theta}^{\mathbf{2}}=\boldsymbol{\theta}^{\mathbf{1}}-\eta^{1} G(\theta)^{1}=\left(\begin{array}{l}2.2 \\ 1.1 \\ 1.1\end{array}\right)$

[Iteration2]: Repeat the rule of [iteration1] but change the sample with another one randomly selected from the sample set.

Assume that the second sample got randomly is sample 2: $\boldsymbol{X}_{\boldsymbol{s}_{1 \times \mathbf{3}}}^{\mathbf{2}}=\left(\begin{array}{lll}\mathbf{2} & \mathbf{2} & \mathbf{1}\end{array}\right), \boldsymbol{F}_{\boldsymbol{s}_{\mathbf{1} \mathbf{1}}}^{\mathbf{2}} \mathbf{1 6}$.

The deviation function is: $V(\theta)_{\mathbf{1} \times \mathbf{1}}^{2}=F_{s}^{2}-F\left(X_{s}^{2}\right)=16-\left(2 \theta_{1}+2 \theta_{2}+\theta_{3}\right)=8.3$

The loss function (Target of the optimization) $J(\theta)_{1 \times 1}^{2}=\frac{1}{2}\left(16-\left(2 \theta_{1}+2 \theta_{2}+\theta_{3}\right)\right)^{2}=34.45$

The gradient of $J(\theta): G(\theta)_{3 \times 1}^{2}=-\left(X_{s}^{2}\right)^{T} V(\theta)^{2}=\left(\begin{array}{c}4 \theta_{1}+4 \theta_{2}+2 \theta_{3}-32 \\ 4 \theta_{1}+4 \theta_{2}+2 \theta_{3}-32 \\ 2 \theta_{1}+2 \theta_{2}+\theta_{3}-16\end{array}\right)=\left(\begin{array}{c}-16.6 \\ -16.6 \\ -8.3\end{array}\right)$

If $\eta^{2}$ is set to be $0.01, \boldsymbol{\theta}^{\mathbf{3}}=\boldsymbol{\theta}^{2}-\eta^{2} G(\theta)^{2}=\left(\begin{array}{l}2.37 \\ 1.27 \\ 1.18\end{array}\right)$, then $J(\theta)^{3}=9.91$

Use the similar way to do following iterations, $J(\theta)^{4}=0.01$ (when $\eta^{3}=0.15$ ).

\section{The Variants of SGD}

In this section, we discuss the variants of SGD. These variants make SGD more useful and can be applied in more settings. There are several techniques for the variants, such as mini-batch, momentum, ADAM and variance reduced method. Then we introduce them below.

Mini-batch. Actually, instead of performing a parameter update for each single training date, usually, we perform the stochastic gradient descent for every mini-batch of n-training data. Formally speaking, it updates as follows.

$$
x^{t+1}=x^{t}-\gamma \nabla \sum_{i=B} f_{i}\left(x^{t}\right)
$$


Where set B includes the training data in a mini-batch. This way reduces the variance of the parameter updates, which can lead to more stable convergence. Moreover, it can make use of highly optimized matrix optimizations that make computing the gradient w.r.t. a mini-batch very efficient. Hence, mini-batch SGD is typically used in machine learning training process.

Take Task 1 as example. Dividing the 4 samples into batch 1 (sample 1 and sample 2) and batch 2(sample 3 and sample 4), then the n (quantity) of training data for each batch is 2 . For the course of solving the Task 1 , the only difference between SGD and mini-batch is that the former uses only one sample while the later uses one batch in each iteration $\boldsymbol{X}_{\boldsymbol{s}}^{\mathbf{1}}{ }_{\mathbf{1} 3}$ and $\boldsymbol{F}_{\boldsymbol{s} \mathbf{1} \times \mathbf{1}}^{\mathbf{1}}$ should be changed to be $X_{s_{2 \times 3}}^{1}=\left(\begin{array}{lll}2 & 1 & 1 \\ 2 & 2 & 1\end{array}\right), F_{s_{2 \times 1}}^{1}=\left(\begin{array}{l}11 \\ 16\end{array}\right), \quad X_{s_{1 \times 3}}^{2}$ and $F_{s_{1 \times 1}}^{2}$ should be changed to be $X_{s_{2 \times 3}}^{2}=$ $\left(\begin{array}{lll}3 & 1 & 1 \\ 3 & 2 & 1\end{array}\right), F_{s_{2 \times 1}}^{1}=\left(\begin{array}{c}14 \\ 19\end{array}\right)$, and there is no the course of random selection in Mini-batch.

Momentum. When the surface curves much more steeply in one dimension than another dimension, SGD has poor performance since it would oscillate across the slopes. A simple way to overcome the weakness is to introduce a momentum term in the update iteration. Momentum simulates the concept inertia in physics. It means that in each iteration, the update mechanism is not only related to the gradient descent, which refers to the dynamic term, but also maintains a component which related to the direction of last update iteration, which refers to the momentum. Since it is similar to push a ball down in hill, the momentum method is also named heavy ball method. Formally, it has the following update rule:

$$
v^{t}=x^{t}-x^{t-1}, \quad v^{t+1}=\rho v^{t}-\gamma \nabla \sum_{i=B} f_{i}\left(x^{t}\right), \quad x^{t+1}=x^{t}+v^{t+1}
$$

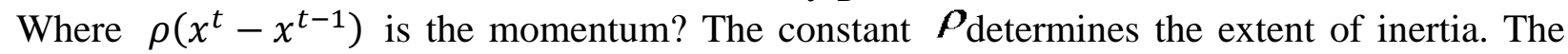
effect of Momentum can be shown in Fig. 2 for an example.

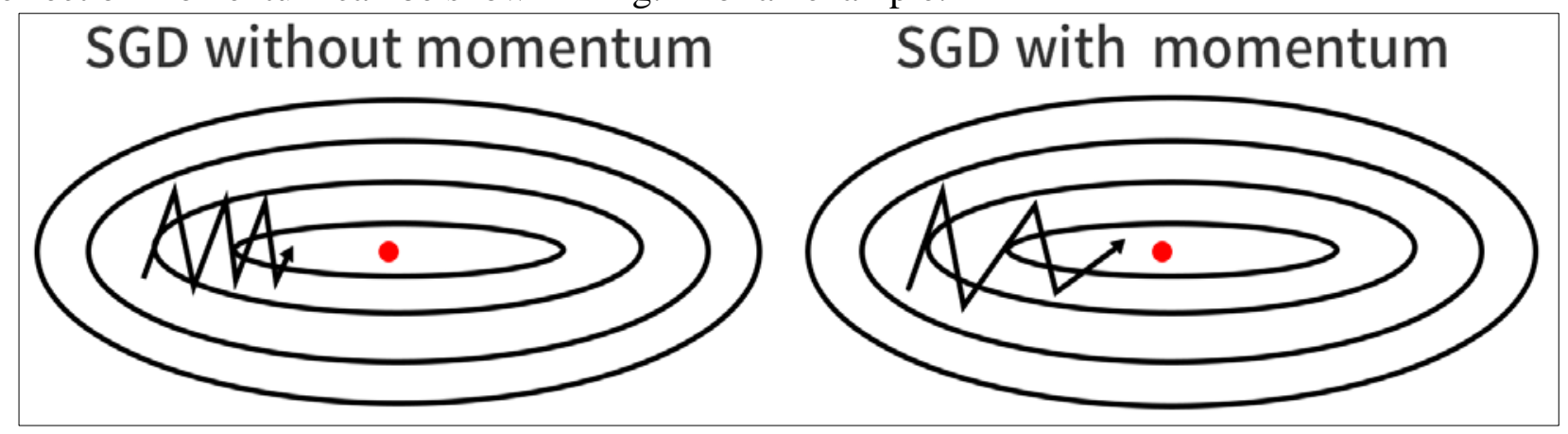

Fig. 2 The compare of the SGD algorithms with and without momentum

Take Task 1 as example. The algorithm in the end of the 3rd iteration of SGD should be changed from $\boldsymbol{\theta}^{\mathbf{3}}=\boldsymbol{\theta}^{\mathbf{2}}-\eta^{2} G(\theta)^{2}=\left(\begin{array}{l}2.37 \\ 1.27 \\ 1.18\end{array}\right)$ to $\boldsymbol{\theta}^{\mathbf{3}}=\boldsymbol{\theta}^{\mathbf{2}}-\eta^{2} G(\theta)^{2}+\rho\left(\boldsymbol{\theta}^{\mathbf{2}}-\theta^{1}\right)=\left(\begin{array}{l}3.47 \\ 1.82 \\ 0.73\end{array}\right)$ (set $\rho=0.5$ ) for Momentum. It shows that the value $\theta^{3}$ approximates to the optimal faster with Momentum. For each of the following iterations in Momentum, $\boldsymbol{\theta}^{\boldsymbol{t + 1}}=\boldsymbol{\theta}^{\boldsymbol{t}}-\eta^{t} G(\theta)^{t}-$ $\rho\left(\theta^{t}-\theta^{t-1}\right)$ is the update rule. According to the experience, $\rho$ is usually set to be $0.5,0.9$ or 0.99 for Momentum.

There are some other ways to choose momentum. Nesterov's momentum is an improvement, which is known as Nesterov's Accelerated Gradient (NAG) descent [13]. The complexity of NAG matches the theoretical lower bound which is $O(1 / \sqrt{\varepsilon})$ for smooth functions and $O(\log (1 / \varepsilon))$ for strongly convex and smooth functions. Start at an arbitrary initial point, NAG iterates the following equations for each step. 


$$
\begin{gathered}
v^{t}=\left(x^{t}-x^{t-1}+\rho v^{t-1}\right) /(1+\rho), v^{t+1}=\rho v^{t}-\gamma \nabla \sum_{i=B} f_{i}\left(x^{t}\right), \\
x^{t+1}=x^{t}-\rho v^{t}+(1+\rho) v^{t+1}
\end{gathered}
$$

There are some works to explain the intuition of NAG and study whether it is the best iterated rules. Recently, Jordan and Al.[19] Show that the Nesterov's momentum has strong relation with the partial differential equations. They show that the trajectory of NAG is corresponding to brachistochrone according to the variational method. See more details for the Nesterov's method, we refer reader to see the survey.[14] Recently, Allen-Zhu provides a new momentum named Katyusha momentum and achieves great performance in many settings.[3]

An illustration of the difference among SGD, Momentum \& Nesterov's momentum is Fig. 3:

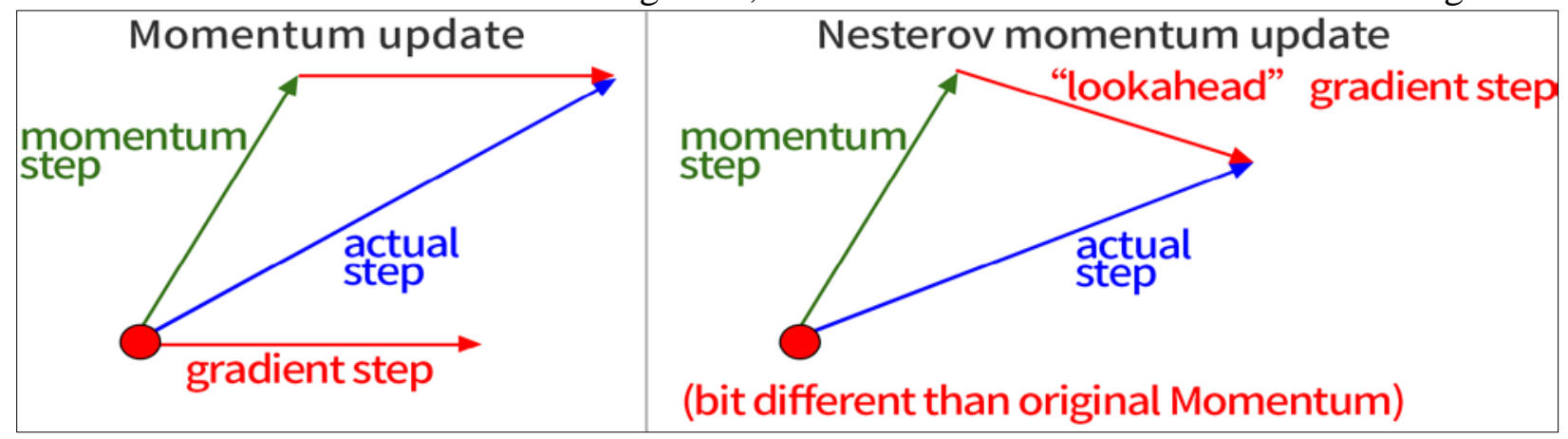

Fig. 3 An illustration of the difference among SGD, Momentum \& Nesterov's momentum

ADAM methods. So far, the method we introduced use the constant step length $\gamma$, which is related to the final computational accuracy. There are some methods to update the step length automatically, such as vSGD, Adadelta, AdaGrad, RMSProp, Adam, Nadam[17][7][9]. AdaGrad adapts the learning rate to the parameters, performing larger updates for infrequent and smaller updates for frequent parameters. Adadelta works well with sparse gradients. RMSProp works well in on-line and non-stationary settings. Adam is designed to combine the advantages of both AdaGrad and RMSProp, which is computationally efficient, has little memory requirements and is well suited for problems that are large in terms of data or parameters.

Then, the update rule of ADAM is as following $\left(a^{b}: b\right.$ is the iteration index No.; $(a)^{b}: b$ is the power):

$$
\begin{aligned}
g^{t+1} & =\nabla f\left(x^{t}\right), m^{t+1}=\beta_{1} m^{t}+\left(1-\beta_{1}\right) g^{t+1}, v^{t+1}=\beta_{2} v^{t}+\left(1-\beta_{2}\right)\left(g^{t+1}\right)^{2}, \\
m^{\prime t+1} & =m^{t+1} /\left(1-\left(\beta_{1}\right)^{t+1}\right), v^{\prime t+1}=v^{t+1} /\left(1-\left(\beta_{2}\right)^{t+1}\right), x^{t+1}=x t-\gamma m^{\prime t+1} /\left(\sqrt{v^{\prime}}+\epsilon\right)
\end{aligned}
$$

Adam is the best proposed algorithm for stochastic optimization and a good default choice for most cases. Show it in detail by taking Task 1 as example here. Set $\alpha=0.001, \beta_{1}=0.9, \beta_{2}=$ 0.999, $\epsilon=10^{-8}, \theta^{1}=0, m^{1}=0, v^{1}=0, \eta^{1}=0.1$ are the initial values, then the algorithm in the end of the 1st iteration of SGD should be changed from $\theta^{2}=\theta^{1}-\eta^{1} G(\theta)^{1}$ to be following [New Iteration1]algorithm:

$$
\begin{gathered}
g^{2}=G(\theta)^{1}=\left(\begin{array}{l}
-22 \\
-11 \\
-11
\end{array}\right), m^{2}=\beta_{1} m^{1}+\left(1-\beta_{1}\right) g^{2}=\left(\begin{array}{l}
-2.2 \\
-1.1 \\
-1.1
\end{array}\right), v^{2}=\beta_{2} v^{1}+\left(1-\beta_{2}\right)\left(g^{2}\right)^{2}= \\
0.726, m^{\prime 2}=\frac{m^{2}}{\left(1-\left(\beta_{1}\right)^{2}\right)}=\left(\begin{array}{c}
-11.58 \\
-5.79 \\
-5.79
\end{array}\right), v^{\prime 2}=\frac{v^{2}}{\left(1-\left(\beta_{2}\right)^{2}\right)}=0.73, \boldsymbol{\theta}^{2}=\boldsymbol{\theta}^{\mathbf{1}}-\frac{\eta^{1} m^{\prime 2}}{\sqrt{{v^{\prime 2}}^{2}}+\epsilon}=\left(\begin{array}{l}
1.36 \\
0.68 \\
0.68
\end{array}\right) .
\end{gathered}
$$

For the following iterations with ADAM, the iterative rules are same.

SVRG. Recently, some researchers find that SGD has slow convergence asymptotically due to the inherent variance. By now, Stochastic Variance Reduced Gradient (SVRG) [8] and its variants such as S2GD, SAG and SAGA [6][10] have delivered much progress through taking advantage of the variance reduced technique. SVRG has linear convergence results for smooth and strongly convex loss. At each time of SVRG, keep a version of estimated $\tilde{\mathrm{x}}$ as that is close to the optimalx. For example, keep a snapshot of $\tilde{\mathrm{x}}$ after every $\mathrm{m}$ SGD iterations. Moreover, maintain the average gradient. $\mu=\frac{1}{n} \sum_{i=1}^{n} \nabla f_{i}(\tilde{x})$. Then, the update rule of SVRG is as follows: 


$$
x^{t+1}=x^{t}-\gamma\left(\nabla f_{i}\left(x^{t}\right)-\nabla f_{i}(\widetilde{x})+\mu\right)
$$

Despite the meteoric rise of VR methods, their analysis for general non-convex problems is largely missing. Recently, there are some works studying to apply SVRG algorithms for the non-convex settings.[16]

\section{Distributed and Non-convex Extension}

So far, we know that SGD is a popular algorithm that can achieve state-of-the-art performance on a variety of machine learning tasks. In the big data era, there are two principle challenges for SGD. One is to apply it in the distributed system. The other is to process the non-convex objective functions, which is common in machine learning problems.

Distributed Extension. Several researchers have recently proposed schemes to parallelize SGD, one of the most famous one is Hogwild![15]. Actually, the algorithm is simply applying SGD in the distributed system without any locking. It means that the algorithm allows processors access to shared memory with the possibility of overwriting each other's work. When the associated optimization problem is sparse, meaning most gradient updates only modify small parts of the decision variable, then Hogwild! Achieves a nearly optimal rate of convergence. Recently, there are some improvement for Hogwild! Besides, there are some works applying the SGD in the dual problem. One of the typical algorithm is parallel dual coordinate descent algorithm [18].

Non-convex Extension. Although, it has achieved great process for the theoretical analysis for SGD recent years, most of the results can only apply for the convex objective functions. As we know, most of the objective functions in machine learning problems are non-convex and SGD works well in practice. To achieve the theoretical guarantee for SGD is the current research focus. There are some partial progresses in this problem. Several works study some special non-convex objective functions and find SGD or its variants can be convergence. Besides, some researchers [2] find that in many machine learning problems, the minimal value of local minimum is a good approximation for the global minimum. Moreover, it is not difficult to obtain a local minimum since the quantity of local minimum is significant. Very recently, there are researchers studying the hitting time [20] for the optimization problems. They find that although, the mixing time (convergence to global minimum) is exponential long in some problems, the hitting time is polynomial. Hence, we can expect to get a good approximation results in polynomial time.

\section{Conclusion}

Optimization problem is the essential problem in many areas such as computer science, physics and economics. As we know, today is the big data era. How to handle the terabyte-class or even petabyte-class datasets is a great challenge for the researchers. In this paper, we introduce the start-of-art algorithm for unconstrained problems in finite-sum form. Moreover, we explain the variants of SGD in details and discuss the two current frontiers about the SGD algorithms.

\section{Reference}

[1] Adler, I., Resende, M. G., Veiga, G., \& Karmarkar, N. (1989). An implementation of Karmarkar's algorithm for linear programming. Mathematical programming, 44(1), 297-335.

[2] Agarwal, N., Allen-Zhu, Z., Bullins, B., Hazan, E., \& Ma, T. (2017, June). Finding approximate local minima faster than gradient descent. In Proceedings of the 49th Annual ACM SIGACT Symposium on Theory of Computing (pp. 1195-1199). ACM.

[3] Allen-Zhu, Z. (2016). Katyusha: The first direct acceleration of stochastic gradient methods. arXiv preprint arXiv:1603.05953.

[4] Bertsekas, D. P. (2011). Incremental gradient, subgradient, and proximal methods for convex optimization: A survey. Optimization for Machine Learning, 2010(1-38), 3. 
[5] Bubeck, S. (2014). Theory of convex optimization for machine learning. arXiv preprint arXiv:1405.4980, 15.

[6] Dantzig, George, Alexander Orden, and Philip Wolfe. "The generalized simplex method for minimizing a linear form under linear inequality restraints." Pacific Journal of Mathematics 5.2 (1955): 183-195.

[7] Defazio, A., Bach, F., \& Lacoste-Julien, S. (2014). Saga: A fast incremental gradient method with support for non-strongly convex composite objectives. In Advances in Neural Information Processing Systems (pp. 1646-1654).

[8] Graves, A., Mohamed, A. R., \& Hinton, G. (2013, May). Speech recognition with deep recurrent neural networks. In Acoustics, speech and signal processing (icassp), 2013 ieee international conference on (pp. 6645-6649). IEEE.

[9] Johnson, R., \& Zhang, T. (2013). Accelerating stochastic gradient descent using predictive variance reduction. In Advances in neural information processing systems (pp. 315-323).

[10]Kingma, D., \& Ba, J. (2014). Adam: A method for stochastic optimization. arXiv preprint arXiv:1412.6980

[11]Konecný, J., \& Richtárik, P. (2013). Semi-stochastic gradient descent methods. arXiv preprint arXiv:1312.1666, 2(2.1), 3.

[12]Liu, D. C., \& Nocedal, J. (1989). On the limited memory BFGS method for large scale optimization. Mathematical programming, 45(1), 503-528.

[13] Nesterov, Y. (1983). A method for unconstrained convex minimization problem with the rate of convergence o(1/k2). Doklady ANSSSR (translated as Soviet.Math.Docl.),vol.269,pp.543- 547

[14] Nesterov, Y. (2013). Introductory lectures on convex optimization: A basic course (Vol. 87). Springer Science \& Business Media.

[15]Recht, B., Re, C., Wright, S., \& Niu, F. (2011). Hogwild: A lock-free approach to parallelizing stochastic gradient descent. In Advances in neural information processing systems (pp. 693-701).

[16]Reddi, S. J., Hefny, A., Sra, S., Poczos, B., \& Smola, A. (2016, June). Stochastic variance reduction for nonconvex optimization. In International conference on machine learning (pp. 314-323).

[17] Tieleman, T., \& Hinton, G. (2012). Lecture 6.5-RMSProp, COURSERA: Neural networks for machine learning. University of Toronto, Tech. Rep.

[18]Tseng, P. (2001). Convergence of a block coordinate descent method for nondifferentiable minimization. Journal of optimization theory and applications, 109(3), 475-494.

[19]Wibisono, A., Wilson, A. C., \& Jordan, M. I. (2016). A variational perspective on accelerated methods in optimization. Proceedings of the National Academy of Sciences, 201614734.

[20]Zhang, Y., Liang, P., \& Charikar, M. (2017). A Hitting Time Analysis of Stochastic Gradient Langevin Dynamics. arXiv preprint arXiv:1702.05575. 